\title{
Translational Medicine Center of West China Hospital
}

\author{
Hongxin Deng ${ }^{*}$, Weimin Li \& Yuquan Wei* \\ West China Hospital of Sichuan University, Chengdu 610041, China
}

Citation: Deng, H., Li, W., and Wei, Y. (2016). Translational Medicine Center of West China Hospital. Sci China Life Sci 59, 1055-1056. doi: $10.1007 / \mathrm{s} 11427-015-4863-\mathrm{y}$

Biotherapy Translational Medicine Center of West China Hospital, Sichuan University is one of the research centers in West China Hospital, which is located in High Tech Zone, Chengdu. It includes several core facilities, such as State Key Laboratory of Biotherapy/one of the National Research and Development (R\&D) Platforms for Novel Drugs, Chengdu National New Drug Pre-Clinical Safety Evaluation Center and GCP Center of West China Hospital. State Key Laboratory of Biotherapy was established by Ministry of Science and Technology of China in 2005, and it became one of the National R\&D Platforms for Novel Drugs founded by Ministries of Science and Technology as well as Public Health in September 2008. It is also supported by national collaborative innovation and it became the collaborative innovation center for biotherapy in April 2013, which is located at Medical Campus of Sichuan University.

Biotherapy Translational Medicine Center of West China Hospital is becoming a highly-regarded, comprehensive and multidisciplinary research center (Figure 1). Through the seamless integration of basic research, preclinical development, translational and clinical medicine, an efficient and fully integrated technology chain for the discovery and development of innovative drug candidates and new therapy has been established in a single institute. One mission of this center is to improve the treatment of major human diseases such as cancer, cardiovascular diseases, obesity, diabetes mellitus, infectious diseases (hepatitis, AIDS, tuberculosis, etc.), inflammatory diseases, neurological disease, as well as chronic autoimmune diseases. Focusing on more than 150 important targets, hundreds of projects for biologic

*Corresponding author (denghongxin01@163.com)

**Corresponding author (email: weiyq@ vip.sina.com) drugs (such as gene and cell therapy, vaccines, monoclonal antibodies, recombinant proteins), synthetic small molecule drugs and small molecule natural products are ongoing.

The center boasts a prominent faculty of nearly 100 well-funded full professors, associate professors and assistant professors with diverse disciplines such as clinical medicine, biotechnology, structural biology, immunology, pharmacology, chemistry, formulations, drug delivery and material sciences. Every year the faculty obtains competitive grants from the government, including the National High Technology Research and Development Program (863 Program) or the Major State Basic Research Development Program (973 Program). Over 400 research papers are published in peer-reviewed international journals every year, including such top journals as New Engl J Med, Nat Rev Drug Discovery, Dev Cell, Nat Chemical Biol, Nature Med, Mol Cell, Proc Natl Acad Sci USA, Cancer Res and Lancet Neurol. To date, the platform has licensed over 150 patents to the commercial sector throughout China. Furthermore, 45 potent candidate drugs, including synthetic and natural small molecular drugs, gene and cell therapy, vaccine, monoclonal antibodies and recombinant proteins, thus far have been transferred to over 30 pharmaceutical companies for commercial development. The candidate drugs are those for the treatment of cancer, diabetes, autoimmune diseases, fibrosis, Hepatitis B/C and HIV.

The platform also provides both key technological services and integral solutions in R\&D for other institutions and companies, including:

(1) Drug target identification and drug screening. The platform has established multiple sub-platforms for R\&D screening of novel molecules, such as genomics, proteomics, 
A

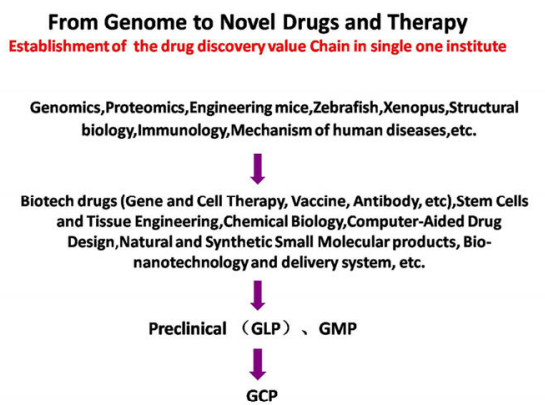

C

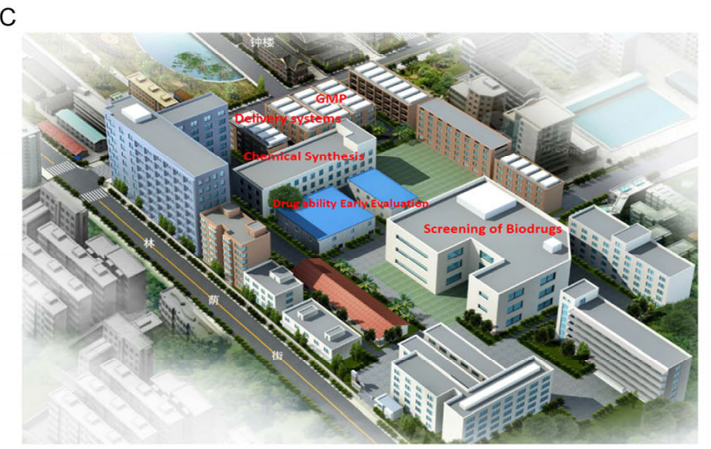

B

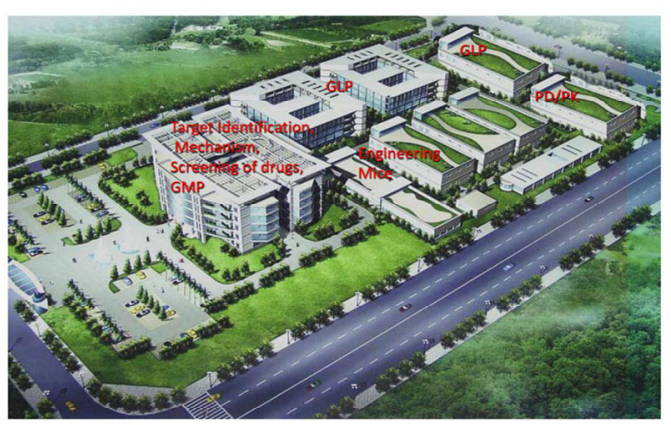

$\mathrm{D}$

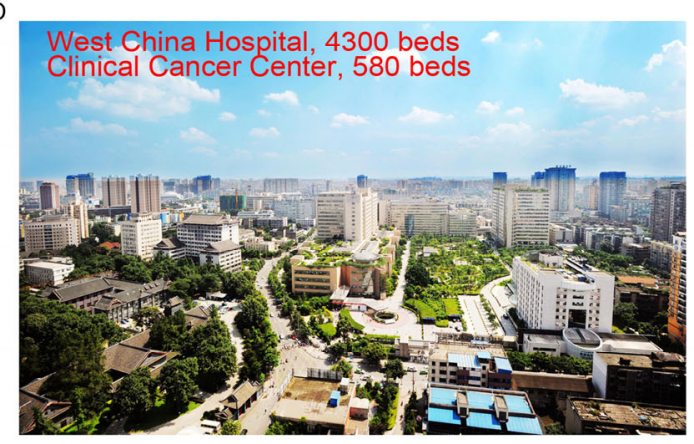

Figure 1 (Color online) Translational Medicine Center of West China Hospital. A, Technical mapping for translational medicine research. B, National Research and Development Platform for Innovative Drugs (Biotherapy Translational Medicine Center of West China Hospital) in High Tech Zone, Chengdu, China. C, National Research and Development Platform for Innovative Drugs (Biotherapy Translational Medicine Center of West China Hospital) in Medical Campus, Chengdu, China. D, Clinical Translational Center of West China Hospital for Innovative Drugs (Biotherapy Translational Medicine Center of West China Hospital) in Chengdu, China.

structural biology, bioinformatics, computer-aided drug design, screening and synthesis of small molecule chemical drugs and the separation and purification of active natural products.

(2) Pilot-scale production. Six GMP-level facilities for pilot-scale production of adenoviral vectors, vaccines, DNA plasmids, recombinant proteins and nanoparticles have been established. For example, the pilot-scale production of DNA plasmids provides 5,000-8,000 doses for early phase clinical trials.

(3) Pharmacodynamics \& pharmacokinetics. Three separate experimental animal facilities can maintain up to 60,000 mice. At full capacity, 300 drug candidates can be evaluated at the same time. To date, there have been over 100 different preclinical disease models established at these facilities.

(4) Pre-clinical safety evaluation. The animal center has currently obtained international "AAALAC" accreditation.
The GLP facility can maintain up to 500 monkeys. Various toxicity studies including single dose toxicity, repeated dose toxicity, reproductive and developmental toxicity, genotoxicity, carcinogenicity, immunogenicity and pharmacokinetic/toxicokinetic (PK/TK) analyses are all performed.

(5) Clinical trials. Clinical trials testing gene and cell therapy, cytokines and anti-cytokines, monoclonal antibodies, chemotherapy and targeted small molecule drugs are conducted in the clinical units of West China Hospital with 4,300 beds.

(6) Graduate programs. The platform also is an educational center. Every year the platform enrolls about 150 Ph.D. and 100 M.S. students for different academic programs, as well as nearly 100 M.S. graduate students for pharmaceutical engineering programs.

Compliance and ethics The author(s) declare that they have no conflict of interest.

Open Access This article is distributed under the terms of the Creative Commons Attribution License which permits any use, distribution, and reproduction in any medium, provided the original author(s) and source are credited. 